\title{
Low energy kinetic threshold in the growth of cubic boron nitride films
}

\author{
S. Kidner, C. A. Taylor II, and Roy Clarke \\ Harrison M. Randall Laboratory of Physics, University of Michigan, Ann Arbor, Michigan 48109-1120
}

(Received 11 November 1993; accepted for publication 16 January 1994)

\begin{abstract}
We report the growth of cubic boron nitride (cBN) films by magnetron sputtering on Si (100) substrates. The films are grown in the presence of negative substrate bias voltages and a nitrogen plasma produced by an electron cyclotron resonance source. We find evidence for a sharp low-voltage threshold in the substrate bias $(-105 \mathrm{~V})$ beyond which the samples are predominantly $\mathrm{cBN}$. The structural quality of the $\mathrm{cBN}$ films is optimized in a narrow range of voltages near this threshold. We discuss the important role of energetic ions in the formation of $\mathrm{cBN}$ in light of recent theoretical findings.
\end{abstract}

The growth of high quality cubic boron nitride (cBN) has been of technological interest for many years. First demonstrated by Wentorf ${ }^{1}$ in $1957, \mathrm{cBN}$ has attractive properties such as extreme hardness, high thermal conductivity, and low chemical reactivity. More recently, $\mathrm{cBN}$ has been gaining attention as a III-V nitride material for semiconductor device applications. The relatively wide band gaps of the III-V nitrides make them particularly well suited for blue to ultraviolet optical devices as well as for high power/high temperature electronic applications. ${ }^{2,3}$

The challenges encountered in growing epitaxial BN films arise in part from the lack of a suitable lattice matched substrate. Perhaps more important is the difference in equilibrium phases for $\mathrm{BN}$ and its III-V nitride counterparts. Unlike the other III-V nitrides, which tend to stabilize in the wurtzitic structure, BN prefers to stabilize in a hexagonal phase (hBN) similar to the graphitic phase of carbon.

The growth of $\mathrm{cBN}$ films has been demonstrated using a variety of deposition processes ${ }^{4-10}$ which all employ an energetic plasma to promote $\mathrm{cBN}$ growth. From these investigations it is clear that the kinetics of the plasma plays an important role in the synthesis of cBN. In this report we present new results of BN films deposited on (100) silicon by rf sputtering using both a low energy electron cyclotron resonance (ECR) ion source and negative substrate bias to promote $\mathrm{cBN}$ formation. We find evidence for a very sharp bias voltage threshold above which $\mathrm{cBN}$ is formed. Moreover, the structural quality of the $\mathrm{cBN}$ is optimized near this threshold. The range of bias voltage over which $\mathrm{cBN}$ is formed is much narrower than previous reports in the literature would indicate. ${ }^{5,10}$

Sputtering of a hot pressed hBN target was carried out in a rf magnetron sputter deposition system with a base pressure of $5 \times 10^{-10}$ Torr. The cryopumped ultrahigh vacuum chamber is equipped with an Astex compact ECR source and a $30 \mathrm{kV}$ reflection high energy electron diffraction (RHEED) system.

The substrate oxide layer was removed thermally at base pressure following a modified Shiraki ${ }^{11}$ clean. The samples were brought to the growth temperature of $850^{\circ} \mathrm{C}$ and dcbiased negatively with respect to ground. The magnetron source-to-substrate distance is approximately $20 \mathrm{~cm}$ and the ECR is mounted at $45^{\circ}$ with a source-to-substrate distance of $8 \mathrm{~cm}$. At these distances the substrate surface is completely immersed in the ECR plasma but is well removed from the plasma confinement region of the sputtering source. During growth the chamber pressure was 1.0 mTorr with flow rates of $10 \mathrm{sccm} \mathrm{Ar}$ into the chamber and $10 \mathrm{sccm} \mathrm{N}_{2}$ through the ECR. Both gases were ultrahigh purity $(99.999+\%)$ and were passed through additional point-of-entry purifiers before entering the gas manifold. The samples were examined in situ immediately after growth using RHEED.

With the rf magnetron power fixed at $600 \mathrm{~W}$ and the microwave ECR power fixed at $200 \mathrm{~W}$, the substrate bias was varied systematically from -100 to $-120 \mathrm{~V}$. With these operating conditions our growth rate is approximately 0.1 $\AA / \mathrm{s}$. Although all of the samples are polycrystalline films there is clear evidence in the RHEED pattern of a sharp threshold in the bias voltage at $-105 \pm 1.0 \mathrm{~V}$ beyond which the samples are cubic. This threshold is revealed by the distinct differences in the RHEED ring patterns of $h B N$ and $\mathrm{cBN}$. Furthermore, the structural quality of the films as monitored by the ring width of the RHEED patterns is optimized in a very narrow window of voltages just beyond the threshold. Figure 1 shows intensity profiles taken along the shadow edge of the RHEED ring patterns for a series of

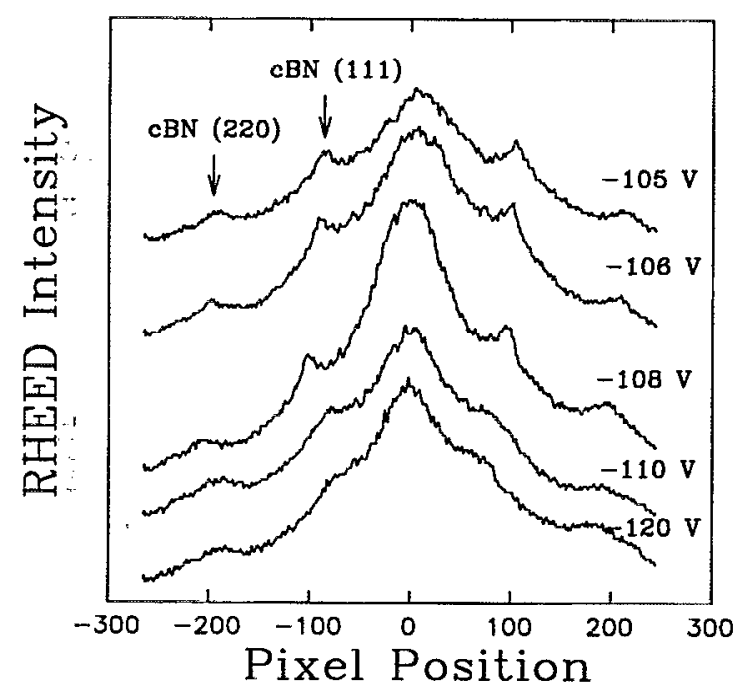

FIG. 1. Intensity profiles of RHEED ring patterns grown near the threshold voltage. The $\mathrm{cBN}$ (111) ring width becomes noticeably sharper and more intense at $-108 \mathrm{~V}$. Beyond $-108 \mathrm{~V}$ the rings quickly broaden. 
TABLE I. RHEED ring width vs substrate bias for samples grown near the threshold voltage. Diffraction ring widths are obtained from fitting the intensity profile to Gaussian line shapes.

\begin{tabular}{lcl}
\hline $\begin{array}{l}\text { Substrate } \\
\text { bias } \\
\text { (V) }\end{array}$ & $\begin{array}{c}\text { Diffraction } \\
\text { ring width } \\
\text { (Pixels) }\end{array}$ & $\begin{array}{c}\text { Structure } \\
\text { and } \\
\text { ring index }\end{array}$ \\
\hline \hline-100 & 51.6 & $\mathrm{hBN}(002)$ \\
-104 & 42.3 & $\mathrm{hBN} \mathrm{(002)}$ \\
-105 & 27.5 & $\mathrm{cBN}(111)$ \\
-106 & 25.2 & $\mathrm{cBN}(111)$ \\
-108 & 23.7 & $\mathrm{cBN} \mathrm{(111)}$ \\
-110 & 38.1 & $\mathrm{cBN} \mathrm{(111)}$ \\
-120 & 57.2 & $\mathrm{cBN}(111)$ \\
\hline
\end{tabular}

samples grown near the threshold voltage. The first set of peaks, to either side of the central diffuse scattering peak, correspond to the cBN (111) diffraction ring. The next set (further out from the diffuse central peak) correspond to the $\mathrm{cBN}(220)$. The width of the cBN diffraction rings is strongly dependent on the bias voltage (see Table I). At the threshold value of approximately $-105 \mathrm{~V}$ the RHEED pattern indicates $\mathrm{CBN}$, while below this value (lcss ncgative bias voltage) only $\mathrm{hBN}$ is formed. In the region between -106 and $-108 \mathrm{~V}$ the rings in the RHEED pattern are much sharper and brighter indicating larger grain sizes and a generally more ordered structure. Beyond $-108 \mathrm{~V}$ the rings begin to noticeably broaden indicating a significant decrease in structural quality.

Shown in Fig. 2 is the infrared spectrum of a $2000 \AA$ film grown at $-108 \mathrm{~V}, 3 \mathrm{~V}$ beyond the threshold. The $\mathrm{cBN}$ TO-mode is observed at the accepted valuc of $1065 \mathrm{~cm}^{-1}$. The hBN $A_{2 u}$ and $E_{1 u}$ modes at 770 and $1380 \mathrm{~cm}^{-1}$ indicate a small amount of hBN in the film. These results are confirmed by plan-view transmission electron microscopy (Fig. 3 ). The diffraction pattern shows sharp rings which index to

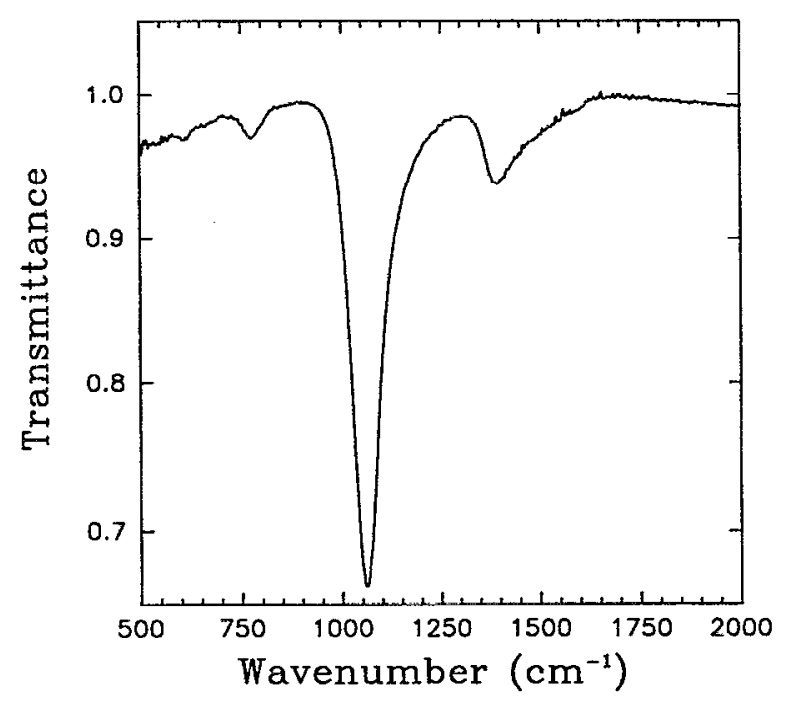

FIG. 2. Infrared spectrum of a cBN film grown with a substrate bias voltage of $-108 \mathrm{~V}$. The $\mathrm{cBN}$ TO mode is at $1065 \mathrm{~cm}^{-1}$ and the small peaks at 770 and $1380 \mathrm{~cm}^{-1}$ indicate a small amount of hBN in the films.

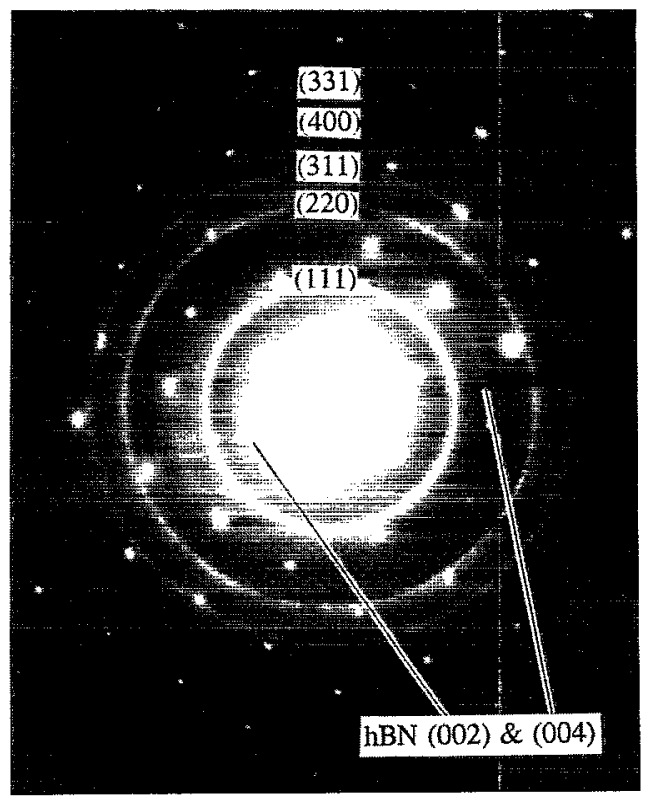

FIG. 3. Plan-view transmission electron diffraction pattern of a BN film grown with a substrate bias voltage of $-108 \mathrm{~V}$. The spot pattern is from the [110] zone of silicon. The very faint and diffuse partial rings index to the $(002)$ and $(004)$ of $\mathrm{hBN}$. All other rings index to $\mathrm{cBN}$.

$\mathrm{cBN}$. The diffuse partial rings in the pattern are due to the small amount of hBN in the film. This small fraction of $h B N$ is not detectable in the RHEED diffraction pattern.

The existence of some type of threshold or range of parameters for $\mathrm{CBN}$ formation has been reported in several investigations utilizing plasma assisted deposition, ${ }^{5-7,9,10}$ nearly all of which have employed high energy ion bombardment from a Kaufman-type source to control the formation of cBN. Using ion bombardment of this type the window for cBN formation, whether it be defined in terms of a substrate bias, ${ }^{5,10}$ an ion energy, ${ }^{6,7,11,12}$ or momentum/atom, exists over a wide range of the controlling parameter. Studies in the literature have shown $\mathrm{cBN}$ formation with ion energies as high as $7 \mathrm{keV}$ with a typical window of formation on the order of hundreds of eV. Several investigations have claimed optimized growth with $\mathrm{N}_{2}$ /Ar ion beams with ion energies of $500 \mathrm{eV}^{6,9,12}$ Our finding, to the best of our knowledge, is the first evidence of a sharp low energy (on the order of $100 \mathrm{eV}$ ) threshold.

The existence of an energy threshold for $\mathrm{cBN}$ formation can be understood by considering how BN transforms from the thermodynamically stable hexagonal structure to the higher energy cubic structure. In general, this transformation requires a hybridization change from $s p^{2}$ to $s p^{3}$ accompanied by a reduction of the interplanar spacing which cannot be accomplished without both bond breaking and a change in the chemical nature of the bond. It is clear that without energetic species, the BN film will stabilize in the hexagonal structure on substrates other than diamond and $\mathrm{cBN}$ itself. While it has been suggested ${ }^{12}$ that $\mathrm{cBN}$ formation is the result of the buildup of biaxial film stress, our findings suggest that the role of the energetic ions is to break the bonds of 
surface atoms and provide the kinetics necessary for cBN formation.

Although our threshold energy is much lower and sharper than previous work would indicate, it is surprising that film growth is possible in the presence of such high kinetic energies. In most material systems collision energies in this range would cause considerable lattice damage. To understand why $\mathrm{cBN}$ can withstand such energies the case of diamond (a similar $s p^{3}$ bonded material) provides considerable insight. Recent theoretical investigations of radiation damage in diamond have shown that the impulse energy required to permanently displace a single atom on the surface of a diamond lattice is approximately $50 \mathrm{eV} .{ }^{13}$ In terms of an incident ion of similar mass this would correspond to kinetic energies in the range of $100 \mathrm{eV}$ which is strikingly similar to our findings. ${ }^{14}$ The authors attribute this extremely high damage threshold in diamond (approximately 3.5 times the cohesive energy) to the large speed of sound in diamond. In effect, there is a rapid dissipation of kinetic energy into the surrounding crystal via incoherent lattice vibrations, in agreement with values derived from experiments using high energy electron bombardment of diamond. ${ }^{15}$

In light of our experimental findings and the recent theoretical work, we conclude that $\mathrm{cBN}$ formation requires the arrival of energetic species on the order of $100 \mathrm{eV}$ to facilitate bond breakage of surface atoms during growth. We expect this energy value to be strongly dependent on deposition rate for a given incident flux of ions since at some point the deposition rate will saturate the kinetic effects provided by the ions and $\mathrm{hBN}$ will be formed. Once this point is reached either the ion flux must be increased or the energy must be increased to provide additional collision kinetics. We believe this may account for the widely varying energy ranges reported in the literature for $\mathrm{CBN}$ growth. Furthermore, our work suggest that an extremely slow deposition rate combined with ions on the order of $100 \mathrm{eV}$ will optimize not only the fraction of $\mathrm{cBN}$ formation but the crystal quality as well.

The authors thank S. Fahy and G. Doll for many valuable discussions regarding our findings. This work was supported by ONR Grant No. N0014-91-J-1398. The authors gratefully acknowledge the encouragement and support of Max Yoder.

${ }^{1}$ R. H. Wentorf, J. Chem. Phys. 26, 956 (1957).

${ }^{2}$ R. F. Davis, Proc. IEEE 79, 702 (1991).

${ }^{3}$ S. Strite and H. Morkoç, J. Vac. Sci. Technol. B 10, 1237 (1992).

${ }^{4}$ M. Mieno, T. Yoshida, and K. Akashi, J. Jpn. Inst. Metals 52, 199 (1988).

${ }^{5} \mathrm{M}$. Mieno and T. Yoshida, Surf. Coatings Technol. 52, 87 (1992).

${ }^{6}$ T. Wada and N. Yamashita, J. Vac. Sci. Technol. A 10, 515 (1992).

${ }^{7}$ N. Tanabe, T. Hayashi, and M. Iwaki, Diamond and Related Materials, 1, 883 (1992).

${ }^{8}$ A. K. Ballal, L. Salamanca-Riba, G. L. Doll, C. A. Taylor, and R. Clarke, J. Mater. Res. 7, 1618 (1992).

${ }^{9}$ D. J. Kester, K. S. Ailey, R. F. Davis, and K. L. More, J. Mater. Res. 8, 1213 (1993).

${ }^{10}$ A. Weber, et al., J. Phys. III (Paris) 2, 1931 (1992).

${ }^{11}$ A. Ishizaka and Y. Shiraki, J. Electrochem. Soc. 133, 666 (1986).

${ }^{12}$ D. Kester and R. Messier, Mater. Res. Soc. Symp. Proc. 35, 721 (1992); J. Appl. Phys. 72, 504 (1992).

${ }^{13}$ W. Wu and S. Fahy, Phys. Rev. B (in press).

${ }^{14}$ The $100 \mathrm{eV}$ energy range assumes that the incident ion will recoil from the lattice upon collision, transferring approximately $50 \%$ of its kinetic energy.

${ }^{15}$ C. D. Clark and E. W. J. Mitchell, Institute of Physics Conference Series (Institute of Physics, London, 1977), p. 45. 